\title{
Optical phonon decay in bulk aluminum nitride
}

\author{
D. Y. Song and M. Holtz ${ }^{\text {a) }}$ \\ Department of Physics, Texas Tech University, Lubbock, Texas 79409
}

A. Chandolu and S. A. Nikishin

Department of Electrical Engineering, Texas Tech University, Lubbock, Texas 79409

E. N. Mokhov, Yu. Makarov, and H. Helava

The Fox Group, Inc., 331 Magnolia Avenue, Piedmont, California 94610

(Received 6 March 2006; accepted 31 May 2006; published online 10 July 2006)

We report Raman studies of the $E_{2}{ }^{2}$ and $\mathrm{A}_{1}(\mathrm{LO})$ symmetry phonons of bulk AlN from 13 to $375 \mathrm{~K}$. Based on observed dependences of the phonon energies and linewidths, and accounting for the temperature-dependent thermal expansion, we determine that both phonon lifetimes are limited by two-phonon decay. The $E_{2}{ }^{2}$ decays symmetrically. The $\mathrm{A}_{1}(\mathrm{LO})$ asymmetrically decays into vibrations with energies 586 and $309 \mathrm{~cm}^{-1}$ which we attribute to $\mathrm{A}_{1}(\mathrm{TO})$ and LA phonons from the $M$ point of the Brillouin zone. () 2006 American Institute of Physics. [DOI: 10.1063/1.2219092]

Wide band gap semiconductors GaN and AlN are receiving great attention due to their importance in deep ultraviolet optoelectronic and high power electronic devices. Because bulk crystals are not widely available, basic and applied studies of these materials have primarily relied on heteroepitaxy using foreign substrates. ${ }^{1,2}$ These epitaxial crystals are generally abundant in dislocations, which strongly affect the optical and electrical properties. Recent advances in growing bulk AlN, with relatively low defect density, present the opportunity to study intrinsic fundamental properties.

It is important to study the vibrational properties of materials particularly for device structures where high current densities produce substantial Joule heating. ${ }^{3}$ This is particularly the case in polar materials, where the Fröhlich electronphonon interaction is dominant. Scattered electrons predominantly emit zone-center longitudinal-optic (LO) phonons. ${ }^{4}$ To dissipate energy from the immediate region, these LO phonons must decay into traveling acoustic waves. The intrinsic phonon properties of high-quality crystalline materials are thus of relevance to self-heating and any phonon engineering efforts to mitigate the associated device problems.

Phonon lifetimes in ideal crystals are determined by decay processes. ${ }^{5}$ Fundamentally, phonons decay into two or more phonons subject to energy and wave vector conservation. The most probable decay channels are those for which the vibrations created have high density of states. The twophonon decay mechanisms are referred to as Ridley and Klemens processes. ${ }^{5,6}$ In the Klemens process an optical phonon decays into two identical acoustic phonons with opposite momenta. The decay of an optical phonon into one transverse optical (TO) phonon and one longitudinal acoustic (LA) phonon is known as the Ridley channel. The twophonon process does not fully describe the phonon decay mechanism in all cases. Under these circumstances, it is usual to include three or higher-order phonon decay processes.

Temperature dependent Raman scattering measurements are ideally suited for examining zone-center phonon decay processes. Under the best conditions, both the phonon energy and line width may be accurately described according to

\footnotetext{
a) Author to whom correspondence should be addressed; electronic mail: mark.holtz@ttu.edu
}

phonon decay. In this letter, we report studies of the $E_{2}{ }^{2}$ and $\mathrm{A}_{1}(\mathrm{LO})$ phonons in bulk AlN. This material is seen to exhibit very narrow Raman bands allowing investigation of the linewidth and its implications in phonon lifetime.

AlN ingots were grown by sublimation sandwich technique described elsewhere. ${ }^{7}$ The ingots were sliced and the Al-faced (0001) oriented samples polished. X-ray and Raman measurements were carried out on free-standing samples with diameter of $1.25 \mathrm{~cm}$ and thickness of $0.3 \mathrm{~mm}$. $\mathrm{X}$-ray diffraction studies are performed using a highresolution diffractometer in double- and triple-axis alignments with $\mathrm{Cu} K \alpha_{1}$ monochromatic radiation. The illumination area is $\sim 1 \times 6 \mathrm{~mm}^{2}$. The $a$ and $c$ lattice constants

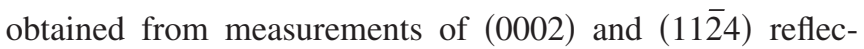
tions are consistent with relaxed AlN material. X-ray $\omega$-scan linewidths were $\sim 25 \operatorname{arcsec}$ for (0002) diffraction and $\sim 38$ arcsec for the $(11 \overline{2} 4)$ diffraction. These compare well with other bulk AlN materials. ${ }^{8,9}$ Micro-Raman measurements were made using a $488.0 \mathrm{~nm}$ excitation and with the sample temperature controlled using a closed-cycle cryostat. $^{10}$ The reported linewidths are corrected for the $2.0 \mathrm{~cm}^{-1}$ instrumental bandpass of the Raman system.

Figure 1 shows Raman spectra for AlN at temperatures $T=20$ and $300 \mathrm{~K}$. The spectra are dominated by two features at 659 and $891 \mathrm{~cm}^{-1}$, corresponding to the $E_{2}^{2}$ and $\mathrm{A}_{1}(\mathrm{LO})$ phonons, respectively. The line width of the $E_{2}{ }^{2}$ phonon at

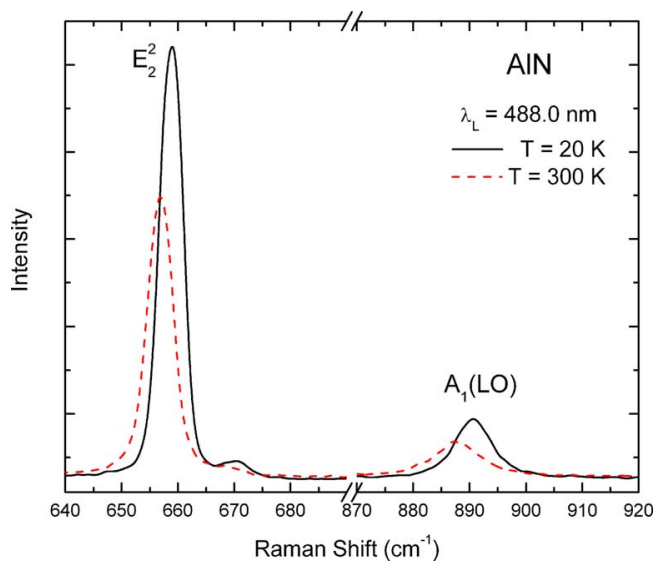

FIG. 1. (Color online) Raman spectra of bulk AlN at two temperatures. 


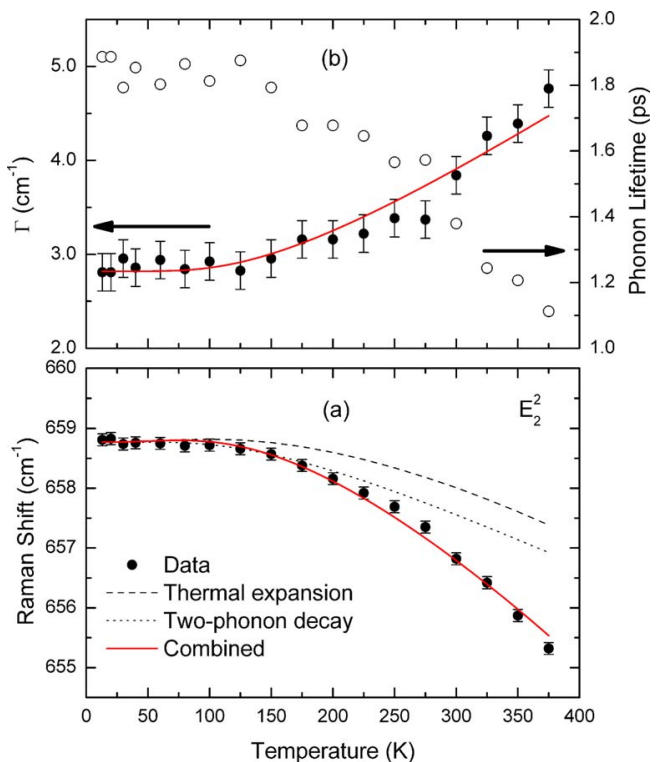

FIG. 2. (Color online) Dependence of $E_{2}^{2}$ phonon energies (a) and linewidths (b) on temperature. Curves are theory-based fits to data. Contributions of thermal expansion and two-phonon decay to the overall shifts are plotted separately. Phonon lifetime as function of temperature is also shown.

$T=20 \mathrm{~K}$ is narrow, comparing well with previously published results ${ }^{11}$ and indicative of high crystal quality. ${ }^{12,13}$ The $\mathrm{A}_{1}(\mathrm{LO})$ phonon observed in our spectrum is likewise very narrow, consistent with high crystal quality and low doping concentration. ${ }^{14}$ The narrow phonon lines are essential for examining of the intrinsic phonon decay properties.

In Figs. 2 and 3 we show the temperature dependences of the phonon energies and line widths for the $E_{2}{ }^{2}$ and $\mathrm{A}_{1}(\mathrm{LO})$, respectively. The Raman shifts decrease and lines broaden with increasing temperature. For bulk materials, temperature dependences of $\omega(T)$ can be described by the thermal expansion and anharmonic (decay) interactions according to

$$
\omega(T)=\omega_{0}-\Delta_{1}(T)-\Delta_{2}(T)
$$

where $\omega_{0}$ is the harmonic frequency of the phonon mode, $\Delta_{1}(T)$ is the thermal expansion contribution to the frequency

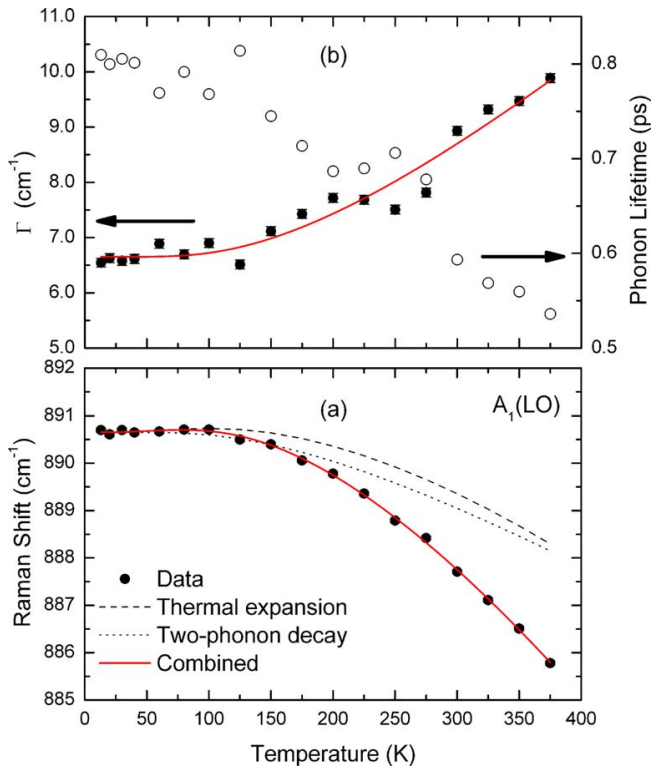

FIG. 3. (Color online) Dependence of $\mathrm{A}_{1}(\mathrm{LO})$ phonon energies (a) and linewidths (b) on temperature. Curves are theory-based fits to data. Contributions of thermal expansion and two-phonon decay to the overall shifts are plotted separately. Phonon lifetime as function of temperature is also shown.
TABLE I. Phonon decay results for AlN phonons. All $T=0 \mathrm{~K}$ values are from fits to the temperature dependences.

\begin{tabular}{lcc}
\hline \hline Phonon & $\begin{array}{c}\text { Energy } \\
\left(\mathrm{cm}^{-1}\right)\end{array}$ & $\begin{array}{c}\text { Linewidth } \\
\left(\mathrm{cm}^{-1}\right)\end{array}$ \\
\hline$E_{2}{ }^{2}$ & $\omega_{0}=661.1$ & $\Gamma_{0}=0.70$ \\
& $A=2.37$ & $C=2.12$ \\
& $\omega_{1}=329.9, \omega_{2}=331.2$ & \\
$\mathrm{~A}_{1}(\mathrm{LO})$ & $\omega_{0}=895.1$ & $\Gamma_{0}=0.93$ \\
& $A=4.49$ & $C=5.73$ \\
& $\omega_{1}=586.1, \omega_{2}=309$ & \\
\hline \hline
\end{tabular}

and $\Delta_{2}(T)$ is the anharmonic interaction term. ${ }^{15}$ The thermal expansion contribution to the shift is given by $\Delta_{1}(T)$ $=\omega_{0} \gamma \int_{0}^{T}\left[\alpha_{c}\left(T^{\prime}\right)+2 \alpha_{a}\left(T^{\prime}\right)\right] d T^{\prime}$ where $\gamma$ is the modeGrüneisen parameter and $\alpha_{c}$ and $\alpha_{a}$ are the temperaturedependent linear thermal expansion coefficients parallel and perpendicular to the hexagonal $c$ axis, respectively. Using published results of $\gamma,{ }^{15} \alpha_{c}$ and $\alpha_{a}{ }^{16} \Delta_{1}(T)$ may be calculated with no fitting parameters, as shown in Figs. 2 and 3.

In the cases where vibrational modes decay via creation of two phonons, the anharmonic contribution to the phonon shift $\Delta_{2}(T)$ is ${ }^{15}$

$$
\Delta_{2}(T)=A\left[1+n\left(\omega_{1}, T\right)+n\left(\omega_{2}, T\right)\right],
$$

where $n(\omega, T)=\left[\exp \left(h c \omega / k_{B} T\right)-1\right]^{-1}$ is the Bose function at energy $h c \omega$. Quantities $\omega_{1}$ and $\omega_{2}$ are frequencies of the phonons created by the decay and $A$ is the anharmonic decay constant. The created/annihilated phonon energies must satisfy conservation of energy. In the cases where the two phonon decay is not sufficient to describe the overall process, higher-order decay terms are included in the expression for $\Delta_{2}(T)$.

In analogy with the above treatment, the linewidth $(\Gamma)$ is described in terms of anharmonic interactions ${ }^{13}$

$$
\Gamma(T)=\Gamma_{0}+\Delta_{2}^{\prime}(T),
$$

where $\Delta_{2}^{\prime}(T)$ is identical in form to $\Delta_{2}(T)$ with coefficients $A$ replaced by $C$. The linewidth is influenced by anharmonic decay and inhomogeneous impurity phonon scattering. Following Ref. 17, measured phonon linewidths may be used to approximate values of the phonon lifetime using ${ }^{11}$

$$
\frac{\Gamma}{\hbar}=\frac{1}{\tau}
$$

where the Planck constant $\hbar=5.3 \times 10^{-12} \mathrm{~cm}^{-1} \mathrm{~s}$ and $\Gamma$ is corrected for the instrumental resolution.

We use Eq. (1) with fitting parameters $A, \omega_{0}, \omega_{1}$, and $\omega_{2}$ to fit the temperature dependence of our $E_{2}^{2}$ Raman shift data, as shown in Fig. 2(a). Fit results are included in each panel and parameters are summarized in Table I. Our analysis shows that the $E_{2}{ }^{2}$ phonon decays primarily by twophonon symmetric decay with identical energies $\omega_{1}=\omega_{2}$ $\sim 330 \mathrm{~cm}^{-1}$. In contrast to $\mathrm{GaN},{ }^{18}$ the Klemens channel fully describes the decay of AlN $E_{2}{ }^{2}$ phonon over this temperature range. In Fig. 2(a), we show the individual contributions of the thermal expansion and two-phonon decay process. These two contributions are found to be comparable across the temperature range examined, illustrating the importance of correctly accounting for the thermal expansion effect. 
Based on the above analysis of the phonon energy, we now analyze the temperature dependence of the $E_{2}^{2}$ linewidth. We use the same value for $\omega_{0}$ obtained previously and fit Eq. (3) to our $E_{2}{ }^{2}$ linewidth data varying parameters $\Gamma_{0}$, $C, \omega_{1}$, and $\omega_{2}$, as shown in Fig. 2(b). Our analysis results in agreement between fitting parameters $\omega_{1}$ and $\omega_{2}$ from Raman shifts and linewidths. Coefficients $A$ and $C$ are likewise in reasonable agreement. Thus, the temperature dependence of the phonon energy and linewidth allow us to obtain consistent results showing the decay of $E_{2}{ }^{2}$ into two phonons with identical energies. Examination of the AlN phonon dispersion curve ${ }^{19}$ suggests that decay into two LA phonons from the $A$ point of the Brillouin zone satisfy the conservation of momentum, although we cannot rule out $M$-point LA phonons.

Figure 3 shows the temperature-dependent Raman shifts and linewidths of the AlN $\mathrm{A}_{1}(\mathrm{LO})$ phonon. The individual contributions from thermal expansion and two-phonon decay to overall Raman shift are separately plotted along with the overall dependence from Eq. (1) with fitting parameters $A$, $\omega_{0}, \omega_{1}$, and $\omega_{2}$. Results are summarized in Table I. Our analysis suggests that the $\mathrm{A}_{1}(\mathrm{LO})$ phonon exhibits asymmetric decay, i.e., $\omega_{1} \neq \omega_{2}$, producing one TO phonon with energy $586 \mathrm{~cm}^{-1}$ and one LA phonon with energy $309 \mathrm{~cm}^{-1}$. Similar to the $E_{2}{ }^{2}$ phonon dependence, the contribution from the two-phonon decay process are comparable in magnitude to that from thermal expansion.

In analogy to our analysis of $E_{2}{ }^{2}$ line width data, we keep the value of $\omega_{0}$ from our temperature dependence of $\mathrm{A}_{1}$ (LO) Raman shift and use Eq. (3) with fitting parameters $\Gamma_{0}, C, \omega_{1}$, and $\omega_{2}$ to fit our $\mathrm{A}_{1}(\mathrm{LO})$ line width data, as shown in Fig. 3(b). Our analysis shows that fitting parameters $\omega_{1}$ and $\omega_{2}$ are consistent, as are the parameters $A$ and $C$. This consistency strongly supports the decay of $\mathrm{A}_{1}(\mathrm{LO})$ phonon into one TO phonon and one LA phonon as the most possible decay channel. From published phonon dispersion curves for AlN, ${ }^{19}$ the created phonons may be attributed to the $\mathrm{A}_{1}(\mathrm{TO})$ and LA vibrations near the $M$ point and possibly the $B_{1}$ and $E_{2}{ }^{1}$ vibrations from the $L$ point of the Brillouin zone. The former decay assignment is the Ridley channel. ${ }^{5}$

In Fig. 2(b), we also plot the temperature dependence of the $E_{2}{ }^{2}$ phonon lifetime calculated using our data and Eq. (4). The $E_{2}{ }^{2}$ phonon lifetime at $20 \mathrm{~K}$ is $1.9 \mathrm{ps}$. Our value of $1.4 \mathrm{ps}$ at $300 \mathrm{~K}$ is close to the value reported in Ref. 11 and slightly larger than that in Ref. 17. The temperature dependence of the $\mathrm{A}_{1}(\mathrm{LO})$ phonon lifetime is plotted in Fig. 3(b). The $\mathrm{A}_{1}(\mathrm{LO})$ phonon lifetime at 20 and $300 \mathrm{~K}$ are 0.8 and $0.6 \mathrm{ps}$, respectively, slightly longer than previous reports. ${ }^{11,17}$

Our decay mechanisms for $E_{2}{ }^{2}$ and $\mathrm{A}_{1}(\mathrm{LO})$ phonons in AlN differ from prior results, in which the $E_{2}{ }^{2}$ phonon asymmetrically decays into two phonons, at 176 and $483 \mathrm{~cm}^{-1}$, and the $\mathrm{A}_{1}(\mathrm{LO})$ phonon decays symmetrically into two phonons at $447 \mathrm{~cm}^{-1}{ }^{11}$ There are several factors which may explain the differences between our results and prior studies. First, we use the temperature dependent thermal expansion coefficients. This factor is important particularly in the temperature range below $\sim 250 \mathrm{~K}$, where $\alpha_{c}$ and $\alpha_{a}$ deviate significantly from the higher-temperature value. Second, we exploit both Raman shifts and linewidths to obtain consistent decay mechanisms based on their temperature dependences for the $E_{2}{ }^{2}$ and $\mathrm{A}_{1}(\mathrm{LO})$ phonons. Finally, III-nitride growth research has resulted in persistent improvements in crystal quality. These improvements permit examination of intrinsic properties and refinement of the important phonon decay mechanisms.

It is interesting to compare phonon decay properties in the III-nitrides. For the $E_{2}{ }^{2}$ phonon in GaN the decay is solely through the three-phonon channel, ${ }^{15,18}$ and for $\mathrm{InN}$ it has been attributed primarily to the three-phonon channel. ${ }^{20,21}$ In contrast, we find the $E_{2}{ }^{2}$ phonon in AlN to decay via the symmetric two-phonon channel. This difference is attributable to the presence of a large gap in the phonon density of states in $\mathrm{InN}$ and $\mathrm{GaN}$, while there is no similar gap for AlN. For the $\mathrm{A}_{1}(\mathrm{LO})$ phonon, GaN exhibits predominantly two-phonon asymmetric decay, ${ }^{15,18}$ with asymmetric two-phonon decay reported for InN (Ref. 21) and a possible contribution from three-phonon symmetric decay. ${ }^{20}$ Consistent with these results, we find the $\mathrm{A}_{1}(\mathrm{LO})$ phonon in AlN to decay by asymmetric two-phonon emission. Thus, over a very large range of cation masses in the wurtzite structure, we conclude that the Ridley decay channel $\mathrm{LO} \rightarrow \mathrm{TO}+\mathrm{LA}$ is valid even in the case of AlN where the gap between optic and acoustic phonon branches is small.

Work at Texas Tech University was supported by the National Science Foundation (Grant Nos. ECS-0323640 and ECS-0304224) and the J. F. Maddox Foundation.

${ }^{1}$ H. Morkoc, J. Mater. Sci.: Mater. Electron. 12, 677 (2001).

${ }^{2}$ N. Grandjean, B. Damilano, and J. Massies, J. Phys.: Condens. Matter 13, 6945 (2001).

${ }^{3}$ I. Ahmad, V. Kasisomayajula, M. Holtz, J. M. Berg, S. R. Kurtz, C. P. Tigges, A. A. Allerman, and A. G. Baca, Appl. Phys. Lett. 86, 173503 (2005).

${ }^{4}$ A. J. Kent and J. K. Wigmore, in Electron-Phonon Interactions in LowDimensional Structures, edited by L. Challis (Oxford University Press, Oxford, 2003), Chap. 2.

${ }^{5}$ B. K. Ridley, J. Phys.: Condens. Matter 8, L511 (1996).

${ }^{6}$ P. G. Klemens, Phys. Rev. 148, 845 (1966).

${ }^{7}$ A. S. Segal, S. Y. Karpov, Y. N. Makarov, E. N. Mokhov, A. D. Roenkov, M. G. Ramm, and Y. A. Vodakov, J. Cryst. Growth 211, 68 (2000).

${ }^{8}$ B. Raghothamachar, M. Dudley, J. C. Rojo, K. Morgan, and L. J. Schowalter, J. Cryst. Growth 250, 244 (2003).

${ }^{9}$ R. Yakimova, A. Kakanakova-Georgieva, G. R. Yazdi, G. K. Gueorguiev, and M. Syvajarvi, J. Cryst. Growth 281, 81 (2005).

${ }^{10}$ M. Holtz, M. Seon, T. Prokofyeva, H. Temkin, R. Singh, F. P. Dabkowski, and T. D. Moustakas, Appl. Phys. Lett. 75, 1757 (1999).

${ }^{11}$ M. Kuball, J. M. Hayes, Y. Shi, and J. H. Edgar, Appl. Phys. Lett. 77, 1958 (2000).

${ }^{12}$ O. Martinez, M. Avella, J. Jimenez, B. Gerard, R. Cusco, and L. Artus, J. Appl. Phys. 96, 3639 (2004).

${ }^{13}$ M. Giehler, M. Ramsteiner, P. Waltereit, O. Brandt, K. H. Ploog, and H. Obloh, J. Appl. Phys. 89, 3634 (2001).

${ }^{14}$ T. Prokofyeva, M. Seon, J. Vanbuskirk, M. Holtz, S. A. Nikishin, N. N. Faleev, H. Temkin, and S. Zollner, Phys. Rev. B 63, 125313 (2001).

${ }^{15}$ A. Link, K. Bitzer, W. Limmer, R. Sauer, C. Kirchner, V. Schwegler, M. Kamp, D. G. Ebling, and K. W. Benz, J. Appl. Phys. 86, 6256 (1999).

${ }^{16}$ K. Wang and R. R. Reeber, Mater. Res. Soc. Symp. Proc. 482, 863 (1998).

${ }^{17}$ L. Bergman, D. Alexson, P. L. Murphy, R. J. Nemanich, M. Dutta, M. A. Stroscio, C. Balkas, H. Shin, and R. F. Davis, Phys. Rev. B 59, 12977 (1999).

${ }^{18}$ D. Y. Song, M. Basavaraj, S. Nikishin, M. Holtz, V. Soukhoveev, A. Usikov, and V. Dmitriev (unpublished).

${ }^{19}$ V. Yu. Davydov, Yu. E. Kitaev, I. N. Goncharuk, A. N. Smirnov, J. Graul, O. Semchinova, D. Uffmann, M. B. Smirnov, A. P. Mirgorodsky, and R. A. Evarestov, Phys. Rev. B 58, 12899 (1998).

${ }^{20}$ X. D. Pu, J. Chen, W. Z. Shen, H. Ogawa, and Q. X. Guo, J. Appl. Phys. 98, 033527 (2005).

${ }^{21}$ J. W. Pomeroy, M. Kuball, H. Lu, W. J. Schaff, X. Wang, and A. Yoshikawa, Appl. Phys. Lett. 86, 223501 (2005). 